CAHIERS DE

NARRATOLOGIE

\section{Cahiers de Narratologie}

Analyse et théorie narratives

8 | 1997

Création de l'espace et narration littéraire

\title{
L'espace narratif dans le Roman de Ferrare de Giorgio Bassani
}

Bernard Urbani

\section{(2) OpenEdition}

1 Journals

Édition électronique

URL : http://journals.openedition.org/narratologie/11735

ISSN : 1765-307X

Éditeur

LIRCES

Édition imprimée

Date de publication : 1 décembre 1997

Pagination : 367-379

ISBN : 291089746X

ISSN : 0993-8516

Référence électronique

Bernard Urbani, «L'espace narratif dans le Roman de Ferrare de Giorgio Bassani », Cahiers de

Narratologie [En ligne], 8 | 1997, mis en ligne le 23 février 2021, consulté le 25 février 2021. URL : http:// journals.openedition.org/narratologie/11735

Ce document a été généré automatiquement le 25 février 2021.

Article L.111-1 du Code de la propriété intellectuelle. 


\title{
L'espace narratif dans le Roman de Ferrare de Giorgio Bassani
}

\author{
Bernard Urbani
}

1 Nourri des grandes traditions littéraires du XIX ${ }^{\text {ème }}$ siècle représentées par Manzoni, Leopardi, Pascoli et influencé par Proust, James et Joyce, Giorgio Bassani, né le 4 mars 1916 à Bologne, est le romancier le plus emblématique de la crise idéale, morale et stylistique que vit la littérature italienne à partir de 1956. A égale distance entre le témoignage historique et la prose d'évasion, Le Roman de Ferrare, composé de six livres ${ }^{1}$, reflète les tourments de l'homme marqué par son destin de victime de l'Histoire. En effet, les lois de discrimination raciale, durement ressenties par les juifs Ferrarais, annoncent la longue période de déportation et d'extermination dont la célèbre plaque commémorative de la Via Mazzini, à Ferrare, est le meilleur des symboles. L'œuvre de Bassani illustre sa hantise du passé qui risque de sombrer à jamais dans l'oubli s'il n'est sauvé grâce à la mémoire. Par des flash-backs, le romancier crée une superposition du passé sur le présent et un contrepoint constant entre un monde révolu et un présent qui donne l'illusion trompeuse de le continuer. Cette reconstruction spatio-temporelle de Ferrare, fortement historicisée, oblige Bassani à se souvenir, à remonter avant 1938 et à écrire une vaste fresque socio-historique de sa ville. Tout au long du Roman de Ferrare, le narrateur recherche dans sa mémoire, comme dans l'Histoire, le passé qui n'est pas définitivement perdu tant que restent tenaces le souvenir et le témoignage. Dans l'immense parc entourant la riche demeure des Finzi-Contini, Micòl, leur fille, a la certitude que seul le passé est encore vivable et constitue un refuge contre l'angoisse d'un présent lourd de menaces et un avenir dont elle pressent inconsciemment le tragique ; en effet, les moments heureux en compagnie du narrateur appartiennent, bel et bien, au «cher, (au) doux, (au) charitable passé »². D'où le désir de tourner le dos à la vie et «d'avancer avec, toujours, la tête en arrière " .

2 Le Roman de Ferrare est «un monument de psychosociologie urbaine et son auteur un entomologiste de la vie citadine $»^{4}$. Bassani décrit sa ville avec ses maisons basses, son château aux tours carrées, image de stabilité, ses palais luxueux et ses jardins mais tout cela ne représente que les grandes lignes d'un décor dont la vérité se situe ailleurs. 
Ferrare, vécue et investie par toutes les pressions sentimentales, sociales et idéologiques que les personnages expérimentent au fil de leurs expériences, devient le tremplin d'une méditation. Le cœur de la cité des Este, jadis prestigieux, et la Piazza del Duomo sont devenus le centre de graves événements, le territoire des fascistes. Le lieu symbolique du pouvoir et de la violence. Dans cette ville "sans qualités", les protagonistes sont déshonorés et condamnés: Bruno Lattès, Geo Josz, l'« unique survivant des 183 membres de la Communauté Israélite que les Allemands avaient déportés dès l'automne $1943 »^{5}$, Clelia Trotti, la socialiste "perdue à jamais dans son solitaire et éternel délire de recluse $»^{6}$, Athos Fadigati, le médecin homosexuel condamné par la société, Micòl Finzi-Contini et toute sa famille, ne sont qu'une caricature face aux « prototypes de la race $»^{7}$. L'immense parc des Finzi-Contini est l'exemple du microcosme figé des Juifs rejetés en marge de la vie dans une existence sans futur. Cette génération perdue, sans défense, égarée par les remous incessants de l'Histoire, pratique une espèce d'autoexclusion en évitant les grandes artères comme, le Corso della Giovecca, et en prenant les ruelles discrètes ou les remparts. Aussi le lieu le plus significatif, à la fois synthèse de géographie et d'histoire, n'est-il pas l'angle entre la Via Vignatagliata et la Via Mazzini là où le comte Scocca, informateur à gages, s'appuie à un "chambranle de marbre qui avait soutenu, pendant des siècles, l'une des trois grilles du ghetto ${ }^{8}$.

3 Si Le Roman de Ferrare se déroule sur fond de guerre et de dictature, cette vision historique traduit une dimension autobiographique confirmée par la présence d'un narrateur qui fait l'expérience d'une plongée dans le passé pour trouver une cause et une justification aux drames qui ont bouleversé son existence. Ce désir d'enraciner les protagonistes est le reflet d'une volonté qui tente de retrouver une mémoire éclatée devenant ainsi un élément majeur dans la construction romanesque. Ce combat contre la fuite du temps se concrétise dans la géographie de Ferrare ${ }^{9}$. Mais Bassani n'est ni Montaigne ni Rousseau et n'a pas écrit de Mémoires d'un Touriste. Son œuvre est un roman original où les éléments puisés dans l'expérience personnelle, selon Dominique Fernandez, "jouent un grand rôle mais après avoir subi une quantité d'amalgames, de déplacements, de transpositions qui constituent le travail créateur $»^{10}$. Certes, le mythe de la capitale des Este se nourrit de la réalité : c'est d'elle qu'il faut partir qu'il s'agisse de l'histoire ou de la géographie. L'espace bassanien signifie toujours « la description ou la représentation verbale d'un lieu physique dont la fonction peut être celle d'éclairer le comportement de personnages romanesques ${ }^{11}$. Il existe, chez le romancier ferrarais, une influence du vécu sur ce qui est écrit mais ce vécu est assimilé, repensé et reconstruit par la mémoire. Saisie par la littérature, revisitée librement, Ferrare devient en partie légendaire et revendique " contre le réalisme qui a toujours fasciné le genre romanesque, les droits de la littérature, et d'abord celui, non de reproduire, mais de produire ${ }^{12}$. Espace réel et romanesque $^{13}$, espace du langage et langage d'un espace, la cité des princes d'Este transcrit des formes et des impressions qui organisent les textes. Sites et paysages ne sont plus des toiles de fond mais sont intimement liés aux événements historiques et au drame personnel de Bassani. L'espace historicisé crée un réseau de lieux de la mémoire qui engendrent « des sentiments d'une intensité éthique considérable, soit dans le registre de la commémoration fervente, soit dans celui de l'exécration, de la déploration, de la compassion, voire de l'appel au pardon $»^{14}$. La masse imposante du Château d'Este, avec ses trois ponts suspendus au-dessus de l'eau vitreuse des fossés, semble, telle une forteresse, commander l'ensemble de la cité à la fois enfermée dans ses remparts et ouverte sur la campagne environnante. Si Bassani n'a pas fait une description détaillée du Corso Ercole d'Este, la plus poétique des rues de Ferrare, la seule dont la physionomie 
originale ait toujours été conservée, il n'en a pas moins souligné la beauté noble de ses édifices et de sa perspective " avec sa lointaine et sublime toile de fond de rouge brique, de vert végétal et de ciel, qui semble vraiment vous conduire vers l'infini $»^{15}$. Ferrare est surtout un enchevêtrement de ruelles médiévales et tortueuses au lourd passé comme, par exemple, la courbe de la célèbre Via Mazzini avec « à son début, l'oratoire de San Maurelio, l'étroite fente de la Via Vittoria en son milieu, la façade en terre cuite rouge un peu plus loin, et la double et parallèle rangée de ses cent magasins et boutiques ${ }^{16}$; les rues fourmillantes d'activités voisinent avec des impasses, à demi désertes, pavées de petits galets blancs et dont les murs s'ornent parfois de coulées de verdure échappées de quelque jardin invisible.

4 La ville, que Bassani a recréée, univers vacillant, ambigu et fluide, revêt une fonction symbolique. Tantôt obstacle, épreuve, exclusion, tantôt lieu d'équilibre propice à la réflexion et à l'apaisement, l'espace ferrarais constitue un enjeu certain; réel et imaginaire, intégré aux personnages comme à l'action et à l'écoulement du temps, il exprime les intentions du romancier. Dans les Histoires de Ferrare, comme dans Le Jardin des Finzi-Contini, dans Les Lunettes d'or comme dans Derrière la porte, la ville, entourée de remparts, évoque un espace totalement fermé, une prison à l'intérieur de laquelle les personnages évoluent sans pouvoir vraiment se situer; les monuments qu'ils rencontrent, comme San Cristoforo, Santa Maria in Vado, Sant'Andrea, le Dôme, le Château, déterminent la structure même des nouvelles et des romans et leur donnent une dimension temporelle. C'est le cas du Corso Giovecca qui, vu en perspective dans La Promenade avant dîner, présente une double construction " en entonnoir "; le lecteur découvre cette artère principale d'après une carte postale mais, cette vue panoramique se restreint rapidement pour ne former qu'un point unique, caractérisé par une jeune fille de vingt ans qui s'éloigne "en suivant le trottoir de gauche du Corso Giovecca, marchant lentement vers l'invisible banlieue de la ville ${ }^{17}$.

5 La géométrie de l'espace urbain sert ici de modèle à celle de l'espace narratif. Le Corso présente deux images opposées : un aspect populaire, celui du XIX ${ }^{\mathrm{ème}}$ siècle provincial et, à partir des années 30 , un aspect cossu, celui d'une grande artère digne des grandes villes italiennes de l'époque. En effet, Gemma Brondi marche le long des trottoirs que bordent des maisons basses et pauvres et s'éloigne du tronçon où se concentre la vie. Or, cette description initiale du lieu est, par avance, révélatrice de la situation qui sera bientôt décrite : mieux, elle conditionne le fonctionnement du récit dans son ensemble. Gemma appartient à une famille paysanne catholique alors qu'Elia Corcos, son époux, est un médecin juif ; son mariage, qui est un échec, la voue à une triste existence dans une maison qui présente elle aussi deux aspects antithétiques : une façade bourgeoise, côté rue, et une façade paysanne, côté jardin. La ville de Ferrare prend alors une valeur de symbole et «témoigne de l'impossible accord entre groupes sociaux différents et de la ségrégation qui s'ensuit » ${ }^{18}$.

6 L'intrigue du Roman de Ferrare et le rythme du récit traduisent la structure de la ville, son foisonnement et ses incohérences. L'espace ferrarais est un lieu conflictuel, un lieu de la déchirure, ouvert et clos, la somme de mille et une situations individuelles et le reflet d'un conflit beaucoup plus vaste. Celui du pouvoir et de l'existence refusée. Celui de deux mondes totalement fermés et opposés dont les Brandi, les Mantovani, les Corcos, les Lattès, les Fadigati, les Finzi-Contini sont la représentation vivante. Lida Mantovani, fille du peuple et catholique, séduite par David Camaioli, jeune bourgeois juif, habite la Via Salinguerra qui se termine au pied des bastions. Mais son bonheur, lié 
au sentiment qu'elle a de conquérir un territoire inconnu se solde par un échec qui la ramène dans cette rue déserte "avec ses petits murs hérissés de tessons de bouteille, ses masures et son pavé à demi recouvert d'herbe " ${ }^{19}$ où elle habite une pièce en sous-sol, une "misérable chambre en parquet poussiéreux et aux deux lits de fer côte à côte, où elle avait passé sa première jeunesse $»^{20}$. Devenue mère et définitivement abandonnée par David, Lida retrouve sa ruelle et son taudis, la misère matérielle et morale. Mariée à l'honnête artisan Oreste Benotti, elle habite une maison excentrée, Viale Cavour, près de la gare ; sa vie monotone de bourgeoise se partage, désormais, entre ce quartier neuf et la Via Salinguerra où son mari possède une boutique. Exclue du cœur de Ferrare, la voilà définitivement marginalisée. Dans Une Plaque Commémorative Via Mazzini, les lieux et les trajets sont eux aussi symboliques. Leurs descriptions illustrent le rapport d'opposition existant entre Geo Josz, un juif Ferrarais, déporté en 1943, de retour des camps de la mort, les fascistes et la bourgeoisie ferraraise. Silencieux et énigmatique, il est encore présent pour rappeler le passé alors que son nom est déjà inscrit avec ceux des autres 183 déportés sur une plaque commémorative Via Mazzini. Geo entre dans une ville réduite à une simple forme géométrique, un «sombre polygone de pierre poussiéreuse " qu'il ne reconnaît plus. C'est dans la Via Mazzini qu'un soir, Geo, que plus personne n'attendait, administre une paire de gifles au comte Scocca, l'ex-informateur de la police fasciste. Alors qu'avec le printemps, la vie renait, ce juif Ferrarais se situe délibérément à contre-courant, revêt les hardes avec lesquelles il est rentré d'Allemagne et s'installe définitivement dans son rôle d'ancien déporté et de survivant indésirable. Il reconquiert autoritairement le Corso Giovecca, le Corso Roma, le Caffè della Borsa, pourchasse ses concitoyens dans les dancings et autres lieux de divertissement où ils oublient les atrocités d'un passé récent : défi constant lancé à une communauté et à une ville dont il incarne la mauvaise conscience. C'est alors que les Ferrarais, corrompus et corrupteurs, le rejettent et l'exilent pour toujours. Dans Les Dernières Années de Clelia Trotti et Une Nuit de 43, Bruno Lattès et Piero Barilari, eux aussi, jugent sévèrement le comportement des Ferrarais qui ont adhéré au fascisme. Bruno, alors qu'il assiste aux funérailles officielles de Clelia Trotti, se souvient de Ferrare remplie de haine et de sang; il se rappelle la marche à travers la ville qui l'a conduit, pour la première fois, jusqu'au 36 Via Fondo Banchetto, le domicile de l'institutrice surveillée par l'O.V.R.A ${ }^{22}$ et leurs dernières promenades. Itinéraire symbolique qui passe par Via Mazzini, Via Saraceno, Via Borgo di Sotto, Via Belfiore, Piazza Santa Maria in Vado et qui correspond à un autre parcours plus intellectuel et plus engagé : en effet, Bruno Lattès, qui traverse une période de crise, puise dans le sentiment de différence une humiliation exaltée et ressent la nécessité de rejoindre les antifascistes et donc de créer un espace nouveau symbole de pureté, de jeunesse et d'éternité. Toutefois, il est conscient de son illusion; il est aussi conscient du désespoir du narrateur et de Micòl Finzi-Contini qui se manquent et passent à côté l'un de l'autre parce qu'ils ne peuvent assumer leur situation de la même manière. Bruno comprend l'isolement des Finzi-Contini dans la "Magna Domus », oasis de lumière au cœur des ténèbres de la ville. En effet, leur grand parc et leur riche demeure représentent, pour Bassani et pour le narrateur, une sorte de sur-espace qui feint le monde, une île qui permet la survie, un Éden encore possible. Mais l'univers «fmzi-continico » délimite à son tour un autre espace clos à l'intérieur duquel s'organise la vie d'une famille entourée par un halo de tendresse désespérée. Ce nouvel espace enferme les FinziContini dans un univers dont la figure est celle de la sphère parfaite qui gravite sur ellemême. Sitôt franchie l'enceinte du «Barchetto del Duca », le narrateur a été fasciné par 
le pouvoir rassurant d'un monde à part sur lequel les événements extérieurs n'ont aucune prise si ce n'est sous la forme d'une angoisse qui l'isole davantage.

7 Le Roman de Ferrare révèle toute une série de personnages reclus et exclus. Ils habitent tous des espaces-prisons qui ouvrent sur le néant. Le pharmacien Barilari, un des personnages d'Une Nuit de 43, paralysé, ne quitte plus sa petite chambre mais il a vu, une nuit de 1943, onze Ferrarais fusillés en représailles après l'assassinat d'un haut dignitaire fasciste : onze corps trouvés sur le trottoir bordant le fossé du Château des Este. Le récit de Bassani laisse entendre que ce carnage a été absurde, que les victimes ont été choisies par hasard et que la ville entière, au courant de ce qui se préparait, n'est pas intervenue. Le procès, inutile, renvoie à nouveau dans l'espace réduit du pharmacien cloué sur son fauteuil. Mais, Barilari « déplace » cet espace qui pourrait le condamner en affirmant qu'il dormait lors de la fusillade. Celui que tout le monde soupçonne est acquitté parce que l'unique témoin ne veut pas reconnaître qu'il a vu, en même temps que l'assassin, à l'autre bout de la place, sa propre femme revenant, comme chaque nuit, d'une aventure galante. La géographie spatiale cède ici à l'Histoire et l'espace au temps : en effet, enfermés dans le labyrinthe de leur ville fortifiée, les personnages sont aussi enfermés dans la prison du temps, nécessairement passé, où le futur est impossible et où le présent existe à peine. On retrouve la symbolique de la prison dans tous les livres du Roman de Ferrare: une prison, reflet d'une topographie limitée, ceinte de bastions, de murs et de maisons où vit une communauté condamnée par la politique, la religion, l'hypocrisie, la lâcheté et le conformisme. En effet, à Ferrare, la peur du scandale corrompt tous les rapports humains, ruine les carrières, empêche les démocrates de s'organiser et amène finalement les fascistes au pouvoir ; à Ferrare, " tout est permis pourvu qu'on soit respectable: les gens n'existent pas en eux-mêmes mais par ce qu'ont en dit $»^{23}$. Prisonniers donc de l'espace séparateur et du temps négateur, ces victimes se réfugient dans le passé. L'idéal, pour elles, seraient plutôt d'abolir le temps, comme le suggère Bassani, par l'intermédiaire d'Elia Corcos à la fin de La Promenade avant dîner: " Il regardait devant lui, sans ne plus voir ni rien ni personne. C'était certes un regard singulier (...). Comme si, à dater justement de l'aube de ce jour-là, il eût toujours $v u$ ainsi les êtres et les choses: c'est-à-dire de haut et comme hors du temps ${ }^{24}$. Abolir le temps, c'est substituer à son image linéaire une image circulaire, voir dans le présent la réactualisation d'événements passés. A la conception d'un temps qui s'écoule et qui use, se superpose, dans Le Roman de Ferrare, une conception répétitive de l'Histoire : la Ferrare d'après-guerre n'est-elle pas l'image de celle d'avant le fascisme ou même de celle pendant la guerre? Bassani, comme ses protagonistes, recherche une sorte d'éternité dans un temps circulaire qui l'enferme dans son propre passé douloureux. Ainsi, son œuvre révèle le double encerclement des personnages qu'elle met en scène car «si nous parlons de cercle du temps, il est possible d'utiliser la même figure géométrique pour traduire l'idée d'emprisonnement dans l'espace, de clôture et de cloisonnement à l'intérieur de la ville $»^{25}$.

8 La ville de Ferrare fonde le lieu bassanien ${ }^{26}$ et donne à la fiction romanesque l'apparence de la réalité. C'est bien un lieu vivant qui «proclame l'authenticité de l'aventure par une sorte de reflet métonymique qui court-circuite la suspicion du lecteur ${ }^{27}$ : rues, boulevards, places, églises, palais et châteaux «se personnalisent en empruntant leur originalité aux êtres avec lesquels ils se trouvent associés tout comme les êtres tirent de ces lieux une profondeur ou une poésie supplémentaires, une dimension de plus " ${ }^{28}$. Épisodes et drames construisent leur propre espace qui est celui de l'œuvre d'art. Le temps a pris la forme de l'espace; le cercle des murs de Ferrare, comme le cercle du temps, remplit une 
double fonction : emprisonner et exclure. Cet aspect carcéral et interdit de la ville obsède Bassani en raison des événements qui ont marqué sa vie et éliminé celle de nombreux Juifs. Celle des Finzi-Contini, par exemple, «tous déportés en Allemagne au cours de l'automne $43 »^{29}$. Le narrateur, devenu adulte, revoit encore le cimetière juif de Ferrare avec ses grands prés parsemés d'arbres, ses tombeaux, ses cippes groupés le long des murs d'enceinte et de séparation et surtout la tombe monumentale de la famille Finzi-Contini.

Pour Bassani, le drame de la cité des princes d'Este est celui d'une ville qui, malgré la richesse de son passé et la beauté de son site, est condamnée à un immobilisme irrémédiable. Face aux persécutions, aux souffrances et à la mort, Ferrare demeure identique à elle-même, inhumaine, hypocrite et violente. Mais les jugements de Bassani, pour sévères qu'ils soient, sont dépouillés de toute passion raciale, politique et religieuse. Le romancier ne se pose pas en moraliste mais en observateur intègre. Sa mémoire remonte le temps; elle recompose des intrigues et des personnages à partir d'une analyse des souvenirs d'enfance et des faits graves et douloureux qui ont marqué la population juive. Bassani, à la manière de Proust, veut retrouver la réalité d'un temps passé composé d'événements, de sensations et de sentiments vécus. Le recul du temps lui permet, non seulement de le clarifier en le passant au filtre de son esprit critique, mais aussi de l'enrichir de toute sa fantaisie de romancier et de poète. Pour Bassani, le temps de la fiction est tourné vers l'avenir mais il est surtout traversé, comme dans A la Recherche du Temps Perdu, de moments rétrospectifs privilégiés auxquels seul l'Art peut donner une seconde vie.

10 Ferrare est une ville réelle, dangereuse et fabuleuse, présente et passée, aimée et détestée que Bassani crée inlassablement. Une ville entre l'imaginaire et le réel. Un espace qui se présente comme "crédible et comme fictif ou du moins non vérifiable $»^{30}$. Une cité qui s'inscrit dans l'économie de l'œuvre unique de Bassani, travaillée, transformée et «produite» par l'écriture. Ferrare : une ville tremplin d'une méditation. Celle du narrateur et de l'auteur qui, comme celui de A la Recherche du Temps Perdu, passe du rêve à la désillusion puis à la transfiguration par la Littérature. Bassani, grâce à la mémoire, reprend possession de ses moments et de ses lieux perdus, leur donne un nouveau souffle et construit un espace qui subit un traitement original : contrairement au roman traditionnel, qui élabore, de façon mimétique, un espace adapté aux exigences de l'action, l'œuvre narrative bassanienne se caractérise par une volonté totalisatrice conduisant à la construction d'un kaléidoscope d'images dont la juxtaposition vise à exprimer l'essence de la réalité ferraraise avec ses personnages, leurs psychologies et leurs drames. La distinction des lieux, des moments et des états psychiques crée une unité dans laquelle des éléments différents, voire opposés, se trouvent organisés, comparés et assimilés. Le Roman de Ferrare est bien une œuvre ancrée dans des circonstances événementielles particulières qui s'efforce de dresser un inventaire des lieux de mémoire significatifs du passé ferrarais et même du passé italien. Elle offre au lecteur, non pas un espace singulièrement vague, morcelé, dénudé et dépeuplé mais un paysage composite fait d'Histoire, d'histoires, de fictions et de symboles. Dès lors, la première fonction de Ferrare, en tant qu'espace, est celle de support de la mémoire collective. L'essentiel du roman bassanien n'est pas uniquement écrire et décrire pour se souvenir mais plutôt apprendre et comprendre

11 Ainsi, placé à la convergence de l'Histoire, de l'imaginaire et de la poésie, axes autour desquels tourne toute l'œuvre de Bassani, Ferrare, ville close dans le temps comme 
dans l'espace, lieu de mémoire et véritable champ de batailles, apparaît comme une voie privilégiée d'accès au fonctionnement du texte, à sa signification idéologique et à son authenticité. Sa première fonction est celle de support de la mémoire collective. Lire Le Roman de Ferrare, c'est se promener dans la ville de Bassani, espace circulaire fermé, c'est "avancer par des mouvements en arrière» et y suivre l'histoire d'une communauté et celle d'une vocation. Lire ses récits, c'est se souvenir, re-parcourir ce long et obscur couloir au fond duquel il y a la vie. La vraie, l'éternelle, aussi vivante que jadis.

\section{NOTES}

1. Quatre romans et deux recueils de nouvelles : Les Lunettes d'or (1958), Le Jardin des Finzi-Contini (1962), Derrière la porte (1964), Le Héron (1968), Histoires de Ferrare (1956, L'Odeur du foin (1972), traduits en français et édités aux éditions Gallimard à partir de 1962.

2. Le Jardin des Finzi-Contini, Paris, Gallimard, 1988, coll. « Folio », n 634, p. 373.

3. Ibid., p. 285.

4. André Sempoux, " $E^{\prime}$ proprio lei la stessa che ritorna in pressoché tutti i miei libri », in, Les Lettres Romanes, Université de Louvain, 1985, tome XXXIX, n 1-2, p. 119.

5. Une Plaque Commémorative Via Mazzini, in Histoires de Ferrare, Paris, Gallimard, 1988, coll. « Folio », n 1984, p. 131.

6. Les Dernières Années de Clelia Trotti, in ibid., p. 251.

7. Ibid.

8. Une Plaque Commémorative Via Mazzini, in Histoires de Ferrare, op. cit., p. 162

9. Pour Bassani, l'espace ferrarais constitue son unique raison d'être. Rappelons que «la représentation de l'espace dans le roman ne constitue qu'un point particulier du problème de la mimésis sur lequel écrivains, historiens et critiques s'acharnent depuis Platon et qui montre sur quelle ambigüité repose toute pratique littéraire » (Roland Bourneuf et Réal Ouellet, L'Univers du Roman, Paris, P.U.F., 1972, coll. « Littératures Modernes ", p. 122.).

10. L'Arbre jusqu'aux racines, Paris, Grasset, 1972, p. 300.

11. Michaël Issacharoff, L'Espace de la Nouvelle, Paris, Corti, 1976, p. 14.

12. Le Roman au XXème siècle, Paris, Belfond, 1990, p. 127.>

13. La mémoire bassanienne est au coeur de la fiction romanesque et en prise directe avec l'Histoire. Le Roman de Ferrare affirme la vanité du romancier d'utiliser l'apport de l'Histoire tout en gardant la liberté d'invention et de sélection. Exemples : le Barchetto del Duca, à l'extrémité du Corso Ercole d'Este, n'existe pas ; quant au jardin des Finzi-Contini, il a été inspiré à Bassani par un très beau parc situé à Ninfa, un bourg du Latium.

14. Paul Ricoeur, Temps et Roman, Paris, Seuil, 1985, coll. "Points-Essais », n²29, tome III, Le Temps Raconté, p. 339.

15. Le Jardin des Finzi-Contini, op. cit., p. 17.

16. Une Plaque Commémorative Via Mazzini, in Histoires de Ferrare, op. cit., p. 134.

17. La Promenade avant dîner, in ibid., pp. 86-87.

18. Marie-Anne Rubat du Mérac, « Dentro le mura di Giorgio Bassani », in Mélanges offerts à Madame le Professeur Maryse Jeuland, Aix-en-Provence, Université de Provence, 1983, p. 196.

19. Lida Mantovani, in Histoires de Ferrare, op. cit., pp. 32-33. 
20. Ibid.

21. Une Plaque Commémorative Via Mazzini, in ibid., p. 139.

22. Organisation de Vigilance et de Répression de l'Antifascisme, la Gestapo italienne.

23. Dominique Fernandez, Introduction à Les Lunettes d'or et autres Histoires de Ferrare, op. cit., p. 11.

24. La Promenade avant dîner, in ibid., p. 128.

25. Marie-Anne Rubat du Mérac, "Dentro le mura di Giorgio Bassani", in Hommage à Madame le Professeur Maryse Jeuland, op. cit., p. 205. Dans L'Odeur du foin, le dernier tome du Roman de Ferrare, Bassani racontant la genèse de ses nouvelles, emploie les termes de 'sphère', de 'cercle', de 'cycle' et affirme, dans Là-bas, au fond du couloir, qu'il a joué et qu'il continue "à jouer avec des formes géométriques en majorité sphériques, cônes, tronc de cônes, cercles concentriques " (Paris, Gallimard, 1996, coll. « L'Etrangère », pp. 151-154).

26. Rappelons que pour Henri Mitterand, "c'est le lieu qui fonde le récit, parce que l'événement a besoin d'un 'ubi' autant que d'un 'quid' ou d'un 'quando' " (Le Discours du Roman, Paris, P.U.F., 1980, p. 194).

27. Ibid.

28. Georges Poulet, L'Espace Proustien, Paris, Gallimard, 1982, coll. « Tel », n 68, pp. 147-148.

29. Le Jardin des Finzi-Contini, op. cit., p. 20.

30. Jean-Paul Goldenstein, Pour lire le roman, Bruxelles, Duculot, 1983, p. 96.

\section{AUTEUR}

\section{BERNARD URBANI}

Université d'Avignon 